\title{
K-SPACES AND BOREL FILTERS ON THE SET OF INTEGERS
}

\author{
JEAN CALBRIX
}

\begin{abstract}
We say that a countable, Hausdorff, topological space with one and only one accumulation point is a point-space. For such a space, we give several properties which are equivalent to the property of being a k-space. We study some free filters on the set of integers and we determine if the associated point-spaces are k-spaces or not. We show that the filters of Lutzer-van MillPol are k-filters. We deduce that, for each countable ordinal $\alpha \geq 2$, there exists a free filter of true additive class $\alpha$ (Baire's classification) and a free filter of true multiplicative class $\alpha$ for which the associated point-spaces are $\mathrm{k}$-spaces but not $\aleph_{0}$, the existence being true in the additive case for $\alpha=1$. In particular, we answer negatively a question raised in J. Calbrix, C. R. Acad. Sci. Paris 305 (1987), 109-111.
\end{abstract}

\section{RAPPELS, NOTATIONS}

1.1. Filtres libres sur l'ensemble des entiers. On note $\omega$ l'ensemble des entiers naturels. Un filtre $\mathcal{F}$ sur l'ensemble des entiers est dit libre si $\bigcap \mathcal{F}=\emptyset$. Le filtre $\mathcal{N}$ des parties cofinies de $\omega$ est appelé filtre de Fréchet. C'est le filtre le moins fin parmi les filtres libres et un filtre $\mathcal{F}$ est libre ssi $\mathcal{N} \subset \mathcal{F}$.

En identifiant une partie de $\omega$ à son indicatrice, un filtre $\mathcal{F}$ sur $\omega$ apparait comme une partie de l'espace métrisable compact $\{0,1\}^{\omega}=2^{\omega}$ dit espace de Cantor.

Pour les boréliens de $2^{\omega}$, on utilise la classification de Baire (voir [1], [3], [6]), et pour tout ordinal dénombrable $\alpha$, on note $\mathbf{A}_{\alpha}$ (resp. $\mathbf{M}_{\alpha}$ ) la classe additive (resp. multiplicative) d'ordre $\alpha$. De même, on note $\mathbf{F}_{\alpha}$ (resp. $\mathbf{G}_{\alpha}$ ) les classes engendrées par les fermés (resp. les ouverts).

Un élément $\beta$ de $2^{\omega}$ est noté $\left(\beta_{0}, \beta_{1}, \ldots\right)$. Pour tout entier $n$, on pose $\beta_{\mid n}=$ $\left(\beta_{0}, \ldots, \beta_{n}\right)$. L'ensemble des suites finies de 0 et de 1 est noté $S$. Soit $s, s^{\prime} \in S$ et $\beta \in 2^{\omega}$. On écrit $s \subset s^{\prime}$ et $s \subset \beta$ si $s^{\prime}$ et $\beta$ prolongent $s$. Pour tout $s \in S$, on note $I(s)$ l'ensemble des $\beta \in 2^{\omega}$ tels que $s \subset \beta$. Naturellement, les $I(s)$ forment une base de la topologie de $2^{\omega}$.

Pour tout $x \in\{0,1\}$, on pose $\bar{x}=1-x$. Pour tout $s=\left(s_{0}, s_{1}, \ldots, s_{n}\right) \in S$, on pose $\bar{s}=\left(s_{0}, s_{1}, \ldots, s_{n-1}, \overline{s_{n}}\right)$.

1.2. Espaces-point. Comme dans [2], nous dirons qu'un espace topologique $X$ est un espace-point si $X$ est un espace topologique, strictement dénombrable, séparé, n'ayant qu'un seul point d'accumulation (que l'on note $\infty$ ). On identifie $X \backslash\{\infty\}$

Received by the editors December 3, 1993.

1991 Mathematics Subject Classification. Primary: 03E15, 04A15, 54-05, Secondary 54C35.

Key words and phrases. Borel filters, point-spaces, k-spaces, $\aleph_{0}$-spaces. 
avec l'ensemble $\omega$ (muni de la topologie discrète). L'ensemble des traces des voisinages (ouverts) de $\infty$ sur $\omega$ est un filtre libre $\mathcal{F}$ sur $\omega$ caractérisant $X$, ainsi l'espace $X$ sera noté $X_{\mathcal{F}}$.

Soit $X$ un espace topologique séparé. Rappelons que $X$ est un $k$-espace si toute partie de $X$ de traces compactes sur les ensembles compacts est un fermé de $X$. L'espace $X$ est dit de Fréchet si pour toute partie $A \subset X$ et tout $x \in \bar{A}$ il existe une suite $\left(x_{n}\right) \subset A$ convergeant vers $x$. Il est connu qu'un espace de Fréchet est un k-espace. L'espace $X$ est dit $\aleph_{0}$ s'il est régulier et s'il existe une suite $\left(A_{n}\right)$ de parties de $X$ telle que pour toute partie compacte $K$ et tout ouvert $G$ contenant $K$, il existe $n$ tel que $K \subset A_{n} \subset G$ (E. Michael).

Nous dirons qu'un filtre libre $\mathcal{F}$ sur $\omega$ est un k-filtre si l'espace $X_{\mathcal{F}}$ est un kespace. De même, nous dirons que $\mathcal{F}$ est $\aleph_{0}$ si l'espace $X_{\mathcal{F}}$ est $\aleph_{0}$.

Pour un espace-point $X_{\mathcal{F}}$, nous dirons qu'une partie $F$ de $X_{\mathcal{F}}$ est du deuxième type si le point $\infty$ est point d'accumulation de $F$. Evidemment, une partie compacte de $X_{\mathcal{F}}$ est du deuxième type ssi elle est infinie.

\subsection{Définition de certains filtres.}

1.3.1. Filtres de type $\mathcal{A}$.

Soit $X$ un espace métrisable séparable possédant une partie strictement dénombrable, dense, constituée de points isolés (que l'on identifie à $\omega$ ). On pose $A=X \backslash \omega$. L'espace quotient obtenu en identifiant les points de $A$ est un espace-point $X_{\mathcal{F}}$ que nous dirons de type $\mathcal{A}$ (Arhangel'skii, cf. [2]). Le filtre $\mathcal{F}$ sera dit aussi de type $\mathcal{A}$. Les filtres de type $\mathcal{A}$ sont des k-filtres, $\aleph_{0}([2])$.

1.3.2. Filtres de type $\mathcal{L} v \mathcal{M} P$.

L'application $\psi: 2^{\omega} \longrightarrow 2^{S}$ définie par $\psi\left(\left(\beta_{0}, \beta_{1}, \ldots\right)\right)=\left\{\left(\beta_{0}\right),\left(\beta_{0}, \beta_{1}\right), \ldots\right\}=\widetilde{\beta}$ est un homéomorphisme de $2^{\omega}$ sur son image. A toute partie $H$ de $2^{\omega}$, correspond une partie $\widetilde{H}=\psi(H) \cup \mathcal{P}_{f}(S)$ où $\mathcal{P}_{f}(S)$ est l'ensemble des parties finies de $S$. Clairement, $\widetilde{H}$ est une sous-base d'un idéal $\mathcal{I}_{H}$ de parties de $S$. Le filtre dual $\mathcal{F}_{H}=\left\{A^{c} / A \in \mathcal{I}_{H}\right\}$ est libre. Il sera dit de type $\mathcal{L} v \mathcal{M P}([1]$, [4], [7]). L'espacepoint associé (en identifiant mentalement $S$ à $\omega$ ) sera noté $X_{H}$ et dit lui aussi de type $\mathcal{L} v \mathcal{M P}$. Nous avons montre dans [1] que pour tout ordinal dénombrable $\alpha \geq 1$, si $H$ est une partie de vraie classe additive $\alpha$, alors il en est de même de $\mathcal{F}_{H}$.

1.3.3. Filtres de type produit et de type $\mathcal{K} a$.

Soit $\left(A_{n}\right)$ une partition de $\omega$ strictement dénombrable constituée de parties strictement dénombrables et soit sur chaque $A_{n}$ un filtre libre $\mathcal{F}_{n}$ que l'on "relève" en un filtre $\widetilde{\mathcal{F}}_{n}$ sur $\omega\left(\widetilde{\mathcal{F}}_{n}=\left\{A \subset \omega / A \cap A_{n} \in \mathcal{F}_{n}\right\}\right)$. Le filtre $\bigcap \widetilde{\mathcal{F}}_{n}$ sera dit du type produit et le filtre $\bigcup_{n} \bigcap_{m \geq n} \widetilde{\mathcal{F}}_{m}$ sera dit du type $\mathcal{K} a$ (Katetov).

Il est montré dans [1] que, pour tout ordinal dénombrable $\alpha$, si les $\mathcal{F}_{n}$ sont de vraie classe $\mathbf{A}_{\alpha}\left(\right.$ resp. $\left.\mathbf{M}_{\alpha}\right), \cap \widetilde{\mathcal{F}}_{n}$ (resp. $\bigcup_{n} \bigcap_{m \geq n} \widetilde{\mathcal{F}}_{n}$ ) est de vraie classe $\mathbf{M}_{\alpha+1}$ (resp. $\left.\mathbf{A}_{\alpha+1}\right)$.

\section{LA PROPRIÉTÉ DE K-ESPACE POUR LES ESPACES-POINT}

La propriété suivante, très simple et de démonstration aisée, sera utile pour la suite :

Lemme 2.1. Un espace-point est un $k$-espace ssi tout fermé du deuxième type contient une partie compacte infinie. En conséquence, un espace-point est un $k$ espace ssi il est de Fréchet. 
Demonstration. Soit $X_{\mathcal{F}}$ un espace-point. Supposons que $X_{\mathcal{F}}$ soit un k-espace. Soit $A$ un fermé du deuxième type. S'il ne contenait pas de partie compacte infinie alors $A \backslash\{\infty\}$ serait fermé, ce qui est absurde. Inversement supposons que $X_{\mathcal{F}}$ ne soit pas un k-espace. Soit une partie $A$ non fermée (donc du deuxième type avec $\infty \notin A$ ) telle que la trace sur elle de toute partie compacte soit compacte. La partie $\bar{A}=A \cup\{\infty\}$ est un fermé du deuxième type qui ne peut évidemment pas contenir une partie compacte infinie.

Notons $A_{\mathcal{F}}$ l'ensemble des ultrafiltres contenant $\mathcal{F}$. L'ensemble $A_{\mathcal{F}}$ est une partie fermée de $\beta \omega$, le compactifié de Stone-Čech de $\omega$. De plus, $A_{\mathcal{F}} \subset(\beta \omega \backslash \omega)$ ssi $\mathcal{F}$ est libre. Rappelons qu'un fermé régulier $A$ d'un espace topologique est un fermé tel $\bar{\circ}$

que $A=A$. Nous obtenons la caractérisation intéressante suivante:

Théorème 2.2. $\mathcal{F}$ est un $k$-filtre ssi $A_{\mathcal{F}}$ est un fermé régulier du sous-espace $\beta \omega \backslash \omega$.

Demonstration. Pour tout $H \subset \omega$, on pose $\widetilde{H}=H \cup\{\infty\}, H^{*}=\bar{H}^{\beta \omega}, H^{* *}=H^{*} \backslash \omega$. Rappelons que, par définition, la collection $\left\{H^{*} / H \subset \omega\right\}$ est une base d'ouverts de la topologie de $\beta \omega$.

Les éléments de $\beta \omega$ seront notés $\mathcal{U}, \mathcal{U}^{\prime}, \ldots$.

FaIt 1. Soit $\mathcal{F}$ un filtre libre. On a $A_{\mathcal{F}}=\{\mathcal{U} / \mathcal{F} \subset \mathcal{U}\}$ est un fermé du sousespace $\beta \omega \backslash \omega$. En effet, $\mathcal{F}$ étant libre, si $\mathcal{F} \subset \mathcal{U}$ alors $\mathcal{U}$ est libre et donc est dans $\beta \omega \backslash \omega$. Soit un ultrafiltre $\mathcal{U}^{\prime}$ tel que on a $\mathcal{U}^{\prime} \notin A_{\mathcal{F}}$. Soit $H \in \mathcal{F} \backslash \mathcal{U}^{\prime}$, donc $\left(H^{c}\right)^{*}$ est un voisinage de $\mathcal{U}^{\prime}$ disjoint de $A_{\mathcal{F}}$.

FAIt 2. Soit $A$ non vide inclus dans $\beta \omega \backslash \omega$. On pose $\mathcal{F}_{A}=\bigcap\{\mathcal{U} / \mathcal{U} \in A\}$. On a $A_{\mathcal{F}_{A}}=\bar{A}$. En effet, $A_{\mathcal{F}_{A}}$ est fermé d'après le fait 1 et contient $A$. Donc $\bar{A} \subset A_{\mathcal{F}_{A}}$. Inversement, soit $\mathcal{U}^{\prime} \notin \bar{A}$ et soit $H \in \mathcal{U}^{\prime}$ tel que $H^{*} \cap A=\emptyset$. Pour tout $\mathcal{U} \in A, H^{c} \in \mathcal{U}$ et donc $H^{c} \in \mathcal{F}_{A}$. Par suite, $H^{c} \in \mathcal{U}$ pour tout $\mathcal{U} \supset \mathcal{F}_{A}$, donc $H^{*} \cap A_{\mathcal{F}_{A}}=\emptyset$ et $\operatorname{donc} \mathcal{U}^{\prime} \notin A_{\mathcal{F}_{A}}$.

En conséquence, l'application de l'ensemble des fermés non vides de $\beta \omega \backslash \omega$ sur l'ensemble des filtres libres de $\omega$ définie par $A \longrightarrow \bigcap\{\mathcal{U} / \mathcal{U} \in A\}$ est une bijection.

FAIT 3. Soit $A$ non vide inclus dans $\beta \omega \backslash \omega$. On a $\mathcal{F}_{A}=\{G \cap \omega / G$ ouvert de $\beta \omega ; G$ $\supset A\}=\{G \cap \omega / G$ ouvert de $\beta \omega ; G \supset \bar{A}\}=\left\{H / H^{*} \supset A\right\}$.

FaIt 4. Soit $A$ non vide, inclus et fermé dans $\beta \omega \backslash \omega$. Soit $K \subset \omega$. La partie $\widetilde{K}$ est une partie compacte infinie de $X_{\mathcal{F}_{A}}$ ssi $K^{* *}$ est non vide et inclus dans $\stackrel{\circ}{A}$. En effet, si $\widetilde{K}$ est une partie compacte infinie de $X_{\mathcal{F}_{A}}$, nécessairement, $K^{* *} \subset A$ et donc $K^{* *} \subset \stackrel{\circ}{A}$. Inversement, si $K^{* *}$ est non vide et dans $\stackrel{\circ}{A}$ et si $F \in \mathcal{F}_{A}$, on a $K \backslash F=K^{*} \backslash F^{*}$ fini et donc $\widetilde{K}$ est une partie compacte infinie de $X_{\mathcal{F}_{A}}$.

Fin de la démonstration du théorème 2.2. Soit $A$ fermé non régulier de $\beta \omega \backslash \omega$. Soit $\mathcal{U}^{\prime} \in A \backslash \stackrel{\bar{\circ}}{A}$. Soit $H \in \mathcal{U}^{\prime}$ tel que $H^{*} \cap \overline{\stackrel{\circ}{A}}=\emptyset$. D'après le fait $4, \widetilde{H}$ est un fermé de $X_{\mathcal{F}_{A}}$ du deuxième type ne contenant pas de partie compacte infinie donc $X_{\mathcal{F}_{A}}$ n'est pas un k-filtre.

Réciproquement, supposons que $X_{\mathcal{F}_{A}}$ ne soit pas un k-filtre. Soit $H \subset \omega$ tel que $\widetilde{H}$ soit un fermé de $X_{\mathcal{F}_{A}}$ du deuxième type ne contenant pas de partie compacte 
infinie. On a $H^{* *} \cap A \neq \emptyset$ mais $H^{* *} \cap \stackrel{\circ}{A}=\emptyset$. Donc $A$ n'est pas un fermé régulier de $\beta \omega \backslash \omega$.

On construit aisément tous les k-filtres:

Proposition 2.3. Soit $\mathcal{K}$ une collection de parties de $\omega$ telle qu'un élément au moins de $\mathcal{K}$ soit infini alors le filtre $\mathcal{F}_{\mathcal{K}}=\{A \subset \omega / \forall K \in \mathcal{K} \quad K \backslash A$ est fini $\}$ est un $k$-filtre et la collection $\{K \cup\{\infty\} / K \in \mathcal{K}\}$ est incluse dans la collection des parties compactes de l'espace-point associé. Inversement, soit $\mathcal{F}$ un filtre libre sur $\omega$ et soit $\mathcal{K}_{\mathcal{F}}$ l'ensemble des traces sur $\omega$ des parties compactes de l'espace-point associé. Si $\mathcal{K}_{\mathcal{F}}$ contient un élément infini alors le filtre $\left\{A \subset \omega / \forall K \in \mathcal{K}_{\mathcal{F}} \quad K \backslash A\right.$ est fini $\}$ est le plus petit $k$-filtre contenant $\mathcal{F}$.

Corollaire 2.4. Avec les notations du théorème précédent, si $\mathcal{K}$ est analytique alors $\mathcal{F}_{\mathcal{K}}$ est co-analytique.

Inversement, soit $\mathcal{F}$ un $k$-filtre et soit $\mathcal{K}_{\mathcal{F}}$ l'ensemble des traces sur $\omega$ des parties compactes de l'espace-point associé. Si $\mathcal{F}$ est analytique alors $\mathcal{K}_{\mathcal{F}}$ est co-analytique. En particulier, si $\mathcal{F}$ et $\mathcal{K}_{\mathcal{F}}$ sont simultanément analytiques alors ils sont boréliens.

La démonstration de la proposition 2.3 est assez directe en utilisant le lemme 2.1 et le corollaire 2.4 en résulte en utilisant le fait que $\left\{(F, K) \in 2^{\omega} \times 2^{\omega} / K \backslash\right.$ $F$ est fini $\}$ est un $F_{\sigma}$.

Les filtres de type $\mathcal{A}$ sont des k-filtres (en effet, l'image quotiente d'un k-espace est un k-espace).

On a aussi:

Théorème 2.5. Les filtres de type $\mathcal{L} v \mathcal{M} P$ sont des $k$-filtres.

Demonstration. Soit $H \subset 2^{\omega}, \mathcal{F}_{H}$ le filtre de type $\mathcal{L} v \mathcal{M} P$ correspondant, et $X_{H}$ l'espace-point associé. Nous allons appliquer le critère du lemme 2.1.

Soit $B \subset X_{H}$ un fermé du deuxième type. Nous dirons qu'une partie infinie $E$ de $B$ est une $H$-chaine maximale s'il existe $\beta \in H$ tel que $E=B \cap \widetilde{\beta}$. On notera $E=q^{\beta}$ et naturellement, $q^{\beta}=\left\{\beta_{\mid n_{0}}, \beta_{\mid n_{1}}, \ldots\right\}$ pour une suite d'entiers strictement croissante.

Ou il n'existe dans $B$ qu'une collection finie $q^{\beta^{0}}, q^{\beta^{1}}, \ldots, q^{\beta^{k}}$ de $H$-chaines maximales alors $B \backslash \bigcup_{i=0}^{k} q^{\beta^{i}}=B \backslash \bigcup_{i=0}^{k} \widetilde{\beta}^{i}$ est une partie compacte du deuxième type.

Ou il existe une infinité de $H$-chaines maximales $q^{\beta^{0}}, q^{\beta^{1}}, \ldots$. Soit $s^{0}=\left(\beta_{0}, \beta_{1}, \ldots\right.$, $\left.\beta_{p_{0}}\right)$ dans $S$ telle que $\left(\beta_{0}, \beta_{1}, \ldots, \beta_{p_{0}}\right) \subset \beta^{k}$ pour une infinité de $k$ et $\overline{s^{0}} \subset \beta^{k_{0}}$ pour au moins un $k_{0}$. Choisissons $m_{0} \geq p_{0}$ tel que $\overline{s^{0}} \subset\left(\beta_{0}^{k_{0}}, \beta_{1}^{k_{0}}, \ldots, \beta_{m_{0}}^{k_{0}}\right) \in$ $q^{\beta^{k_{0}}} \subset B$. On poursuit la construction par récurrence. Supposons construits les $s^{i}=\left(\beta_{0}, \beta_{1}, \ldots, \beta_{p_{0}}, \ldots, \beta_{p_{i}}\right)$ et les $\left(\beta_{0}^{k_{i}}, \ldots, \beta_{m_{i}}^{k_{i}}\right) \in B$ pour tout $i=0,1, \ldots, r$ avec $p_{0}<p_{1}<\ldots<p_{r}, \overline{s^{i}} \subset\left(\beta_{0}^{k_{i}}, \ldots, \beta_{m_{i}}^{k_{i}}\right) \in q^{\beta^{k_{i}}}$ pour tout $i=0,1, \ldots, r$ et $s^{r} \subset \beta^{k}$ pour une infinité de $k$. On peut alors trouver $s^{r+1}=\left(\beta_{0}, \beta_{1}, \ldots, \beta_{p_{0}}, \ldots, \beta_{p_{r+1}}\right)$ tel que $p_{r+1}>p_{r}, s^{r+1} \subset \beta^{k}$ pour une infinité de $k$ et $\overline{s^{r+1}} \subset \beta^{k_{r+1}}$ pour un $k_{r+1}$. On choisit alors $m_{r+1} \geq k_{r+1}$ tel que $\overline{s^{r+1}} \subset\left(\beta_{0}^{k_{r+1}}, \ldots, \beta_{m_{r+1}}^{k_{r+1}}\right) \in q^{\beta^{k_{r+1}}} \subset B$. Il est clair que $K=\left\{\left(\beta_{0}^{k_{0}}, \ldots, \beta_{m_{i}}^{k_{i}}\right) / i \in \omega\right\} \cup\{\infty\}$ est une partie compacte du deuxième type incluse dans $B$. 
Un filtre de type $\mathcal{K} a$ n'est jamais un k-filtre (les parties compactes de l'espace point associé sont finies). Par contre, un filtre de type produit est un k-filtre ssi les filtres associés $\mathcal{F}_{n}$ sont des k-filtres.

Le filtre $\left\{A \subset \omega / \sum_{n \notin A} \frac{1}{n}<+\infty\right\}$ est un filtre libre $K_{\sigma}$ qui n'est pas un k-filtre.

On déduit de ce qui précède et de [1]:

Théorème 2.6. Pour tout ordinal dénombrable $\alpha \geq 2$, chaque classe $\mathbf{A}_{\alpha} \backslash \mathbf{M}_{\alpha}$ et $\mathbf{M}_{\alpha} \backslash \mathbf{A}_{\alpha}$ contient un $k$-filtre. Il en est de même de la classe $F_{\sigma} \backslash G_{\delta}$. Pour tout ordinal dénombrable $\alpha \geq 1$, chaque classe $\mathbf{F}_{\alpha} \backslash \mathbf{G}_{\alpha}$ contient un filtre libre non $k$-filtre.

\section{LA PROPRIÉTÉ $\aleph_{0}$}

Nous savons que les filtres de type $\mathcal{A}$ sont $\aleph_{0}$. Par ailleurs, il est montré dans [4] que, pour $H=2^{\omega}$, le filtre $\mathcal{F}_{H}$ de type $\mathcal{L} v \mathcal{M} P$ n'est pas $\aleph_{0}$. Nous généralisons ce résultat:

Théorème 3.1. Soit $\mathcal{F}_{H}$ un filtre de type $\mathcal{L} v \mathcal{M} P$. Si $H$ contient un sous-ensemble $C$ homéomorphe à l'espace de Cantor alors $\mathcal{F}_{H}$ n'est pas $\aleph_{0}$.

Demonstration. Pour tout $\beta \in C$ et tout $n \in \omega$, il existe $m>n$ tel que $I\left(\beta_{0}, \ldots, \beta_{m}\right) \cap$ $C \neq \emptyset$ et $I\left(\beta_{0}, \ldots, \beta_{m-1}, \overline{\beta_{m}}\right) \cap C \neq \emptyset$. Cette propriété sera appliquée dans la récurrence qui va suivre.

Soit $\left(A_{n}\right)$ une suite de parties de $2^{S}$ et posons $\widetilde{A}_{n}=A_{n} \cup\{\infty\}$. Soit $s^{0}=$ $\left(\beta_{0}, \ldots, \beta_{n_{0}}\right)$ tel que $I\left(s^{0}\right) \cap C \neq \emptyset$ et si $\overline{s^{0}} \in A_{0}$ alors $s^{0} \in A_{0}$. Supposons construit $s^{k}=\left(\left(\beta_{0}, \beta_{1}, \ldots, \beta_{n_{0}}, \ldots, \beta_{n_{k}}\right)\right.$, on peut trouver $s^{k+1}=\left(\beta_{0}, \ldots, \beta_{n_{0}}, \ldots, \beta_{n_{k}}, \ldots, \beta_{n_{k+1}}\right)$ tel que $n_{k}<n_{k+1}, I\left(s^{k+1}\right) \cap C \neq \emptyset$ et si $\overline{s^{k+1}} \in A_{k+1}$ alors $s^{k+1} \in A_{k+1}$. On constuit ainsi un élément $\beta$ de $2^{\omega}$ dans $C$. Posons $K=\left\{\overline{s^{i}} / i \in \omega\right\} \cup\{\infty\}$. Clairement $K$ est une partie compacte de $X_{H}$ incluse dans $2^{S} \backslash \widetilde{\beta}$ et si $K \subset \widetilde{A}_{k}$ alors $\widetilde{A}_{k} \cap \widetilde{\beta} \neq \emptyset$. D'où $X_{H}$ n'est pas $\aleph_{0}$.

Un filtre de type produit est $\aleph_{0}$ ssi les filtres $\mathcal{F}_{n}$ associés sont $\aleph_{0}$.

En utilisant ce qui vient d'être dit, le fait classique que tout espace analytique non dénombrable contient un Cantor et les résultats de [1], on complète le théorème 2.6:

Corollaire 3.2. Pour tout ordinal dénombrable $\alpha \geq 2$, dans chaque classe $\mathbf{A}_{\alpha} \backslash \mathbf{M}_{\alpha}$ et $\mathbf{M}_{\alpha} \backslash \mathbf{A}_{\alpha}$, il existe un $k$-filtre non $\aleph_{0}$. Il en est de même pour la classe $F_{\sigma} \backslash G_{\delta}$.

Remarques. Dans [2], il est demandé si un k-filtre est automatiquement $\aleph_{0}$. Le corollaire 3.2. répond à cette question par la négative. Subsiste la question : est-ce qu'un k-filtre $\aleph_{0}$ est de type $\mathcal{A}$ ? La possibilité de construire un k-filtre $\aleph_{0}$ qui soit analytique non $F_{\sigma \delta}$ répondrait par la négative à cette question (voir [2]).

\section{BIBLIOGRAPHIQUES}

1. J. Calbrix, Classes de Baire et espaces d'applications continues, C. R. Acad. Sc. Paris, t. 301, série I, n 16, (1985), 759-762. MR 87a:54021

2. J. Calbrix, Filtres sur les entiers et ensembles analytiques, C. R. Acad. Sc. Paris, t. 305, série I (1987), 109-111. MR 88j:54051

3. G. Choquet, Lectures in Analysis, W. A. Benjamin, New York, 1969. MR 40:3252;

4. T. Dobrowolski, W. Marciszewski, J. Mogilski, On topological classification of function spaces $C_{p}(X)$ of low Borel complexity, Trans. Amer. Math. Soc. 328 (1991), 307-324. MR 92c:54017

5. R. Engelking, General topology, PWN, Warzawa, 1976. MR 58:18316b 
6. K. Kuratowski, Topology, PWN, Warzawa, 1966. MR 36:840

7. D. Lutzer, J. van Mill, R. Pol, Descriptive complexity of function spaces, Trans. Amer. Math. Soc. 291 (1985), 121-128. MR 87e:54046

Laboratoire A.M.S. URA C.N.R.S. D1378, U.F.R. Des Sciences, F76821 Mont Saint Aignan cedex, France

E-mail address: Jean.Calbrix@univ-rouen.fr 